\title{
Faire partie de la SCPH - un investissement dans votre avenir et celui de la pharmacie hospitalière!
}

par Glen J. Pearson

Un investissement dans le savoir rapporte toujours les meilleurs intérêts. -Benjamin Franklin

$Q_{\mathrm{s}}^{\mathrm{u}}$ ue signifie l'adhésion à notre association professionnelle, la Société canadienne des pharmaciens d'hôpitaux (SCPH)? Certains membres pourraient ne pas s'être posé cette question cruciale pendant des années, voire jamais. D'autres pèsent le pour et le contre chaque année au moment de renouveler leur adhésion et se demandent comment justifier la dépense. Et vous, considérezvous votre adhésion à la SCPH comme une dépense ou un investissement? Peut-être est-ce les deux.

Le terme " dépense " fait référence aux coûts d'achat d'un produit, d'un service ou d'un avantage. Cette opération simple offre aux membres de la SCPH la chance d'assister à la Conférence sur la pratique professionnelle, de lire le Journal canadien de la pharmacie hospitalière et d'accéder aux publications officielles de la SCPH. De nombreux membres de la SCPH accordent une grande valeur à ces produits et services ainsi qu’à d'autres et l'accès à ceux-ci justifie amplement pour eux les coûts de l'adhésion. Par contre, d'autres pharmaciens argumenteront qu'ils peuvent avoir accès à tout cela (directement ou indirectement) sans être membres. L'adhésion à la SCPH doit donc avoir une valeur supérieure.

Je crois qu'il est nécessaire pour les pharmaciens hospitaliers de considérer sérieusement l'adhésion comme une occasion d'investir, comme une dépense pour laquelle il y a une attente de gains futurs! Bien que de nombreux pharmaciens d'hôpitaux puissent ne jamais avoir songé aux dividendes auxquels ils peuvent s'attendre de l'adhésion à la $\mathrm{SCPH}$, c'est là que réside le réel avantage d'en être membres.

La SCPH apporte un soutien à ses membres au moyen de la valorisation, de la formation, du partage de l'information, de la promotion des meilleures pratiques, de l'appui à la recherche et de la reconnaissance de l'excellence. C'est grâce au développement et à l'intensification de ce travail de la SCPH que chaque membre détient un potentiel de gains substantiels - en d'autres termes, c'est là que se trouvent les futurs dividendes.

Pensez aux efforts considérables de la SCPH en ce qui touche le leadership en valorisation et dans les politiques de la santé. Bien que ce ne soit pas nécessairement apparent pour tous les pharmaciens hospitaliers, la SCPH travaille activement à la valorisation de leur expertise quant à bon nombre de sujets, dont la prescription, la sécurité des patients et les modèles de pratique interprofessionnelle. De plus, la direction et les membres du personnel de la SCPH répondent couramment aux consultations menées par d'autres organismes et le gouvernement fédéral sur les politiques de la santé. Par exemple, nous faisons partie d'un comité directeur multilatéral sur les pénuries de médicaments, nous avons fourni nos commentaires au Groupe consultatif provincial-territorial d'experts sur l'aide médicale à mourir et nous avons préparé une réponse formelle au Comité externe (fédéral) sur les options de réponse législative à Carter c. Canada. De plus, nous entretenons régulièrement différentes directions de Santé Canada de politiques liées à la santé et à la pharmacothérapie qui touchent les pharmaciens hospitaliers, notamment en ce qui a trait aux pénuries de médicaments, aux retraits de médicaments, à la délivrance de marihuana à des fins médicales dans les hôpitaux, à la destruction sur place des stupéfiants et des drogues contrôlées, et à la gestion de la prescription de la méthadone aux patients hospitalisés. Bien que les démarches de la SCPH dans ces domaines puissent, de prime abord, sembler très éloignées des préoccupations quotidiennes de la plupart des pharmaciens hospitaliers, elles sont le produit direct d'enjeux qui touchent ou pourraient influencer le travail des pharmaciens d'hôpitaux. Ces démarches importantes dépendent de votre adhésion.

Lorsque vous investissez dans la Société en devenant membre, vous investissez immanquablement dans l'avenir de chaque praticien en pharmacie hospitalière et dans celui de la profession. Réfléchissez à ces avenirs lorsque vous renouvelez votre adhésion et encouragez vos collègues en pharmacie hospitalière à faire le même investissement. N'oubliez pas que, comme l'a dit l'entraîneur de football américain Vince Lombardi, « les réalisations d'un organisme sont le fruit des efforts concertés de chacun ". Participez à la Société - le rendement de votre investissement croîtra nettement!

[Traduction par l'éditeur]

Glen J. Pearson, B. Sc., B. Sc. Phm., Pharm. D., FCSHP, est président et agent de liaison externe pour la Société canadienne des pharmaciens d'hôpitaux. 